\title{
NON-REAL POLES AND IRREGULARITY OF DISTRIBUTION
}

\author{
DAVID LOWRY-DUDA
}

\begin{abstract}
We study the general theory of weighted Dirichlet series and associated summatory functions of their coefficients. We show that any non-real pole leads to oscillatory error terms. This applies even if there are infinitely many non-real poles with the same real part. Further, we consider the case when the non-real poles lie near, but not on, a line. The method of proof is a generalization of classical ideas applied to study the oscillatory behavior of the error term in the prime number theorem.
\end{abstract}

\section{Overview and Results}

In this article, we study the general theory of Dirichlet series $D(s)=$ $\sum_{n \geq 1} a(n) n^{-s}$ and the associated summatory function of the coefficients, $A(x)=\sum_{n \leq x}^{\prime} a(n)$ (where the prime over the summation means the last term is to be multiplied by $1 / 2$ if $x$ is an integer). For convenience, we will suppose that the coefficients $a(n)$ are real, that not all $a(n)$ are zero, that each Dirichlet series converges in some half-plane, and that each Dirichlet series has meromorphic continuation to $\mathbb{C}$. Perron's formula shows that $D(s) / s$ and $A(x)$ are duals and satisfy

$$
\frac{D(s)}{s}=\int_{1}^{\infty} \frac{A(x)}{x^{s+1}} d x, \quad A(x)=\frac{1}{2 \pi i} \int_{\sigma-i \infty}^{\sigma+i \infty} \frac{D(s)}{s} x^{s} d s
$$

for an appropriate choice of $\sigma$.

Many results in analytic number theory take the form of showing that $A(x)=\operatorname{MT}(x)+E(x)$ for a "Main Term" MT $(x)$ and an "Error Term" $E(x)$. Typically the terms in the main term $\mathrm{MT}(x)$ correspond to poles from $D(s)$, while $E(x)$ is hard to understand. Upper bounds for the error term give bounds for how much $A(x)$ can deviate from the expected size, and thus describe the regularity in the distribution of the coefficients $\{a(n)\}$. In this article, we investigate lower bounds for the error term, corresponding to irregularity in the distribution of the coefficients.

This work was supported by the Simons Collaboration in Arithmetic Geometry, Number Theory, and Computation via the Simons Foundation grant 546235.

The author wants to thank Thomas Hulse, Henryk Iwaniec, Chan Ieong Kuan, and Alexander Walker for their encouraging remarks. 
More generally, we can consider a sequence of denominators $0<\lambda_{1}<$ $\lambda_{2}<\cdots$, where $\lambda_{n} \rightarrow \infty$, and consider the generalized Dirichlet series

$$
D_{\lambda}(s)=\sum_{\lambda_{n}} \frac{a(n)}{\lambda_{n}^{s}}
$$

with the associated summatory function $A^{(\lambda)}(x)=\sum_{\lambda_{n} \leq x}^{\prime} a(n)$. We note that one can assume without loss of generality that $\lambda_{1}=1$ by considering the related Dirichlet series

$$
\lambda_{1}^{s} \sum_{\lambda_{n}} \frac{a(n)}{\lambda_{n}^{s}}=\sum_{\lambda_{n}} \frac{a(n)}{\left(\lambda_{n} / \lambda_{1}\right)^{s}}
$$

and then translating results. Thus we will assume our series have been normalized so that $\lambda_{1}=1$.

Results of the form $A^{(\lambda)}(x)=\mathrm{MT}(x)+E(x)$ for these generalized Dirichlet series are also prevalent in number theory. For example, one can associate an Epstein zeta function of the form $D_{\lambda}(s)$ to any complete lattice $\Lambda \subset \mathbb{R}^{d}$; in this example, $A_{\lambda}$ counts the number of lattice points contained within a ball of a given radius.

Further, to get satisfactory understanding of the error terms, it is often necessary to work with smoothed sums $A_{v}^{(\lambda)}(x)=\sum_{\lambda_{n}} a(n) v\left(\lambda_{n} / x\right)$ for a weight function $v(\cdot)$. In this article, we consider nice weight functions, i.e. weight functions with good behavior and whose Mellin transforms have good behavior. For almost all applications, it suffices to consider weight functions $v(x)$ on the positive real numbers that are piecewise smooth and that take values halfway between jump discontinuities.

For such a weight function $v(\cdot)$, denote its Mellin transform by

$$
V(s)=\int_{0}^{\infty} v(x) x^{s} \frac{d x}{x} .
$$

Then we can study the more general dual family

$$
D_{\lambda}(s) V(s)=\int_{1}^{\infty} \frac{A_{v}^{(\lambda)}(x)}{x^{s+1}} d x, \quad A_{v}^{(\lambda)}(x)=\frac{1}{2 \pi i} \int_{\sigma-i \infty}^{\sigma+i \infty} D_{\lambda}(s) V(s) x^{s} d s .
$$

We prove two results governing the irregularity of distribution of weighted sums. Our first theorem guarantees that a non-real pole of $D_{\lambda}(s) V(s)$ corresponds to an oscillatory error term for $A_{v}^{(\lambda)}(x)$.

Theorem 1. Suppose $D_{\lambda}(s) V(s)$ has a pole at $s=\sigma_{0}+i t_{0}$ with $t_{0} \neq 0$ of order $r$. Let $\operatorname{MT}(x)$ be the sum of the residues of $D_{\lambda}(s) V(s) X^{s}$ at all real poles $s=\sigma$ with $\sigma \geq \sigma_{0}$.

Then

$$
\sum_{\lambda_{n}} a(n) v\left(\frac{\lambda_{n}}{x}\right)-\operatorname{MT}(x)=\Omega_{ \pm}\left(x^{\sigma_{0}} \log ^{r-1} x\right) .
$$

Here and below, we use the notation $f(x)=\Omega_{+}(g(x))$ to mean that there is a constant $k>0$ such that $\lim \sup f(x) /|g(x)|>k$ and $f(x)=\Omega_{-}(g(x))$ 
to mean that $\lim \inf f(x) /|g(x)|<-k$. When both are true, we write $f(x)=$ $\Omega_{ \pm}(g(x))$. This roughly means that $f(x)$ is about as positive as $|g(x)|$ and about as negative as $-|g(x)|$ infinitely often.

Our second theorem concerns the case when there are infinitely many non-real poles very near a line.

Theorem 2. Suppose $D_{\lambda}(s) V(s)$ has at least one non-real pole, and that the supremum of the real parts of the non-real poles of $D_{\lambda}(s) V(s)$ is $\sigma_{0}$. Let $\operatorname{MT}(x)$ be the sum of the residues of $D_{\lambda}(s) V(s) X^{s}$ at all real poles $s=\sigma$ with $\sigma \geq \sigma_{0}$.

Then for any $\epsilon>0$,

$$
\sum_{\lambda_{n}} a(n) v\left(\frac{\lambda_{n}}{x}\right)-\operatorname{MT}(x)=\Omega_{ \pm}\left(x^{\sigma_{0}-\epsilon}\right) .
$$

The idea at the core of these theorems is old, and was first noticed during the investigation of the error term in the prime number theorem. To prove them, we generalize those techniques. In particular, we generalize the proofs given in Chapter 5 of Ingham's 1932 monograph [Ing90] (recently republished). In his monograph, Ingham describes a proof showing that $\psi(x)-x=\Omega_{ \pm}\left(x^{\Theta-\epsilon}\right)$ and $\psi(x)-x=\Omega_{ \pm}\left(x^{1 / 2}\right)$, where $\psi(x)=\sum_{p^{n} \leq x} \log p$ is Chebyshev's second function and $\Theta \geq \frac{1}{2}$ is the supremum of the real parts of the non-trivial zeros of $\zeta(s)$. We note that modern monographs or summaries on topics concerning analysis of $\zeta(s)$ will have these results, but as far as the author knows they are never treated in the same level of generality as we present here.

Thus in this article, we generalize these techniques and extend them to weighted sums and more general Dirichlet series.

\section{Motivation And Application}

Infinite lines of poorly understood poles appear regularly while studying shifted convolution series of the shape

$$
D(s)=\sum_{n \geq 1} \frac{a(n) a(n \pm h)}{n^{s}}
$$

for a fixed $h$. When $a(n)$ denotes the (non-normalized) coefficients of a weight $k$ cuspidal Hecke eigenform on a congruence subgroup of $\operatorname{SL}(2, \mathbb{Z})$, for instance, one can provide meromorphic continuation for the shifted convolution series $D(s)$ via spectral expansion in terms of Maass forms and Eisenstein series. The Maass forms contribute infinite lines of poles.

Explicit asymptotics would take the form

$$
\sum_{n \geq 1} a(n) a(n-h) e^{-n / X}=\sum_{j} C_{j} X^{\frac{1}{2}+\sigma_{j}+i t_{j}} \log ^{m} X
$$


where neither the residues nor the imaginary parts $i t_{j}$ are well-understood. Is it be possible for these infinitely many rapidly oscillating terms to experience massive cancellation for all $X$ ? The theorems above prove that this is not possible.

In this case, applying Theorem 1 with the Perron-weight

$$
v(x)= \begin{cases}1 & x<1 \\ \frac{1}{2} & x=1 \\ 0 & x>1\end{cases}
$$

shows that

$$
\sum_{n \leq X}^{\prime} \frac{a(n) a(n-h)}{n^{k-1}}=\Omega_{ \pm}(\sqrt{X}) .
$$

Similarly, Theorem 2 shows that

$$
\sum_{n \leq X}^{\prime} \frac{a(n) a(n-h)}{n^{k-1}}=\Omega_{ \pm}\left(X^{\frac{1}{2}+\Theta-\epsilon}\right),
$$

where $\Theta<7 / 64$ is the supremum of the deviations to Selberg's Eigenvalue Conjecture (sometimes called the non-arithmetic Ramanujan Conjecture).

More generally, these shifted convolution series appear when studying the sizes of sums of coefficients of modular forms. A few years ago, Hulse, Kuan, Walker, and the author began an investigation of the Dirichlet series whose coefficients were themselves $|A(n)|^{2}$ (where $A(n)$ is the sum of the first $n$ coefficients of a modular form). We showed that this Dirichlet series has meromorphic continuation to $\mathbb{C}$ [HKLDW17], but also that there are lines of poles coming from Maass forms as described above. The behavior of the infinite lines of poles in the discrete spectrum play an important role in the analysis, but we did not yet understand how they affected the resulting asymptotics. The author intends to revisit these results, and others, from the context of this article.

\section{Proofs}

Given a function $f(x)$ that is bounded and integrable over any finite interval, the Dirichlet integral

$$
F(s)=\int_{1}^{\infty} \frac{f(x)}{x^{s+1}} d x
$$

behaves in many ways like a Dirichlet series. Most classical results for Dirichlet series correspond to analogous results for Dirichlet integrals. For instance, one can prove that if a Dirichlet integral converges at $s=\sigma_{1}+i t_{1}$, then it converges for all $s$ with $\operatorname{Re} s>\sigma_{1}$ in the same way one proves it for Dirichlet series, except one replaces summation-by-parts with integrationby-parts. Thus a Dirichlet integral will also have a well-defined abscissa of convergence. 
We will make repeated reference to the following theorem of Landau. It is often presented only for Dirichlet series, but it also is true for Dirichlet integrals.

Theorem 3 (A Theorem of Landau). Suppose that $f(x)$ is bounded, integrable in any finite interval, and of constant sign for all sufficiently large $x$. Then the real part $s=\sigma_{1}$ of the abscissa of convergence of the Dirichlet integral $F(s)$ is a singularity.

Proof. This is Theorem H in [Ing90]. The proof is analogous to proofs for the Dirichlet series version.

Since $D_{\lambda}(s)$ is meromorphic on $\mathbb{C}$ and has a half-plane of convergence, there are at most finitely many real poles in any half-plane $\operatorname{Re} s>\sigma$. Near a pole $s=u$ of order $r, D_{\lambda}(s) V(s)$ has a Laurent expansion

$$
\sum_{m=1}^{r} \frac{c_{m}}{(s-u)^{m}}+\phi_{u}(s)
$$

for an analytic function $\phi_{u}(s)$. Then the residue of $D_{\lambda}(s) V(s) X^{s}$ at $s=u$ can be written as

$$
\sum_{m=1}^{r} \frac{c_{m}}{(m-1) !} X^{m} \log ^{m-1} X
$$

Finally, note that

$$
\int_{1}^{\infty} \frac{(\log x)^{m}}{x^{s+1}} d x=\frac{m !}{s^{m+1}} .
$$

We are now ready to prove the two $\Omega_{ \pm}$theorems.

Proof of Theorem 2. Let $\sigma_{0}$ be the supremum of the real parts of the poles poles $s=\sigma+i t$ with $t \neq 0$ of $D_{\lambda}(s) V(s)$; let $\mathrm{MT}(x)$ denote the sum of the residues at the (finitely many) real poles $s=\sigma+i t$ of $D_{\lambda}(s) V(s) X^{s}$ with $\sigma \geq \sigma_{0}$.

Let $f(x)=A_{v}^{(\lambda)}(x)-\operatorname{MT}(x)$, and consider the Dirichlet integral

$$
F(s)=\int_{1}^{\infty} \frac{f(x)}{x^{s+1}} d x .
$$

For $s$ in the half-plane of absolute convergence of $D_{\lambda}(s) V(s)$, we recognize

$$
F(s)=\int_{1}^{\infty} \frac{A_{v}^{(\lambda)}(x)}{x^{s+1}} d x-\int_{1}^{\infty} \frac{\mathrm{MT}(x)}{x^{s+1}} d x=D_{\lambda}(s) V(s)-(* *)
$$

where $(* *)$ is a sum of terms of the form (2). Thus $F(s)$ has meromorphic continuation to $\mathbb{C}$ and the poles of $F(s)$ are a subset of the poles of $D_{\lambda}(s) V(s)$. At any pole $s=\sigma+i t$ with $\sigma \geq \sigma_{0}$, one can check that the Laurent expansion (1) cancels with the part of $\int_{1}^{\infty} \mathrm{MT}(x) x^{-s-1} d x$ corresponding to that pole. Thus $F(s)$ has no real pole $s=\sigma+i t$ with $\sigma \geq \sigma_{0}$.

Fix any real $k, c$ with $k>0$ and $c<\sigma_{0}$. Define

$$
g(x):=A_{v}^{(\lambda)}(x)-\operatorname{MT}(x)-k x^{c} .
$$


Suppose for the sake of contradiction that $g(x)>0$ for all sufficiently large $x$. Then by Landau's Theorem, we know that the abscissa of convergence of

$$
G(s)=\int_{1}^{\infty} \frac{g(x)}{x^{s+1}} d x=F(s)-\frac{k}{(s-c)}
$$

is real. Denote this abscissa of convergence by $\sigma_{1}$. As $F(s)$ has a non-real pole with real part greater than $\sigma_{0}-\epsilon$ for any $\epsilon>0$, we have that $\sigma_{1} \geq \sigma_{0}$.

On the other hand, any real pole $s=\sigma+i t$ of $G(s)$ with $\sigma \geq \sigma_{0}$ must come from $F(s)$. But we have demonstrated that $F(s)$ has no real poles with real part at least $\sigma_{0}$. By contradiction, we have shown that $A_{v}^{(\lambda)}(x)-\operatorname{MT}(x)=$ $\Omega_{-}\left(x^{c}\right)$ for any $c<\sigma_{0}$. A similar contradiction proves the corresponding $\Omega_{+}$result, concluding the proof.

The proof of Theorem 1 begins in the same way.

Proof of Theorem 1. Let $\sigma_{0}+i t_{0}, t_{0} \neq 0$ denote a non-real pole of $D_{\lambda}(s) V(s)$ of order $r$, and let $\operatorname{MT}(x)$ denote the sum of residues at the real poles $s=$ $\sigma+i t$ with $\sigma \geq \sigma_{0}$. As in the proof of Theorem 2, let $f(x)=A_{v}^{(\lambda)}(x)-\mathrm{MT}(x)$; consider the Dirichlet integral $F(s)$ from (3); it remains true that $F(s)$ has no real poles with real part $\geq \sigma_{0}$.

Let $g_{k}(x)$ denote

$$
g_{k}(x)=A_{v}^{(\lambda)}(x)-\mathrm{MT}(x)-k x^{\sigma_{0}} \log ^{r-1} x
$$

for a real constant $k>0$ to be specified later. Let us suppose that $g_{k}(x)>0$ for all sufficiently large $x$. We will show that taking $k$ sufficiently small will lead to a contradiction.

By Landau's Theorem, we know that the abscissa of convergence, $\sigma_{1}$, of

$$
G_{k}(s)=\int_{1}^{\infty} \frac{g_{k}(x)}{x^{s+1}} d x=F(s)-\frac{k /(r-1) !}{\left(s-\sigma_{0}\right)^{r}}
$$

is real. The pole at $\sigma_{0}+i t_{0}$ guarantees that $\sigma_{1} \geq \sigma_{0}$ (but the pole may correspond to the pole coming from $x^{\sigma} \log ^{r-1} x$ ).

Fix $Y$ such that $g_{k}(x)>0$ for all $x>Y$. For any $\sigma>\sigma_{1}$, we have that

$\left|G_{k}\left(\sigma+i t_{0}\right)\right| \leq \int_{1}^{Y} \frac{\left|g_{k}(x)\right|}{x^{\sigma+1}} d x+\int_{Y}^{\infty} \frac{g_{k}(x)}{x^{\sigma+1}} d x=G_{k}(\sigma)+\int_{1}^{Y} \frac{\left|g_{k}(x)\right|-g_{k}(x)}{x^{\sigma+1}} d x$.

Note also that

$$
\int_{1}^{Y} \frac{\left|g_{k}(x)\right|-g_{k}(x)}{x^{\sigma+1}} d x \leq 2 \int_{1}^{Y} \frac{\left|g_{k}(x)\right|}{x^{\sigma_{0}+1}} d x=K
$$

for a finite constant $K$ that is independent of $\sigma$ and $t$. Thus

$$
\left|G_{k}\left(\sigma+i t_{0}\right)\right| \leq K+G_{k}(\sigma) .
$$

Multiply each side of $(5)$ by $\left(\sigma-\sigma_{0}\right)^{r}$. We will evaluate the limit as $\sigma \rightarrow \sigma_{0}$ from the right. In terms of the Laurent expansions, this has the effect of comparing the (absolute value of the) Laurent coefficients of $\left(s-\left(\sigma_{0}+i t_{0}\right)\right)^{-r}$ in $G_{k}\left(\sigma+i t_{0}\right)$ on the left and of $\left(s-\sigma_{0}\right)^{-r}$ in $G_{k}(\sigma)$ on 
the right. In particular, if we specialize (1) to the pole at $\sigma_{0}+i t_{0}$, then $\lim _{\sigma \rightarrow \sigma_{0}^{+}}\left|G_{k}\left(\sigma+i t_{0}\right)\left(\sigma-\sigma_{0}\right)^{r}\right|=\left|c_{r}\right|$. On the other hand, from (4), we see that $\lim _{\sigma \rightarrow \sigma_{0}^{+}}\left(K+G_{k}(\sigma)\right)=k /(r-1)$ !.

Thus multiplying each side of $(5)$ by $\left(\sigma-\sigma_{0}\right)^{r}$ and taking the limit as $\sigma \rightarrow \sigma_{0}$ from the right gives the inequality

$$
\left|c_{r}\right| \leq \frac{k}{(r-1) !}
$$

But $k$ can be chosen freely, and it is clear that choosing $k<(r-1) !\left|c_{r}\right|$ leads to a contradiction. Thus $g_{k}(x)=\Omega_{-}\left(x^{\sigma_{0}} \log ^{r-1} x\right)$. The $\Omega_{+}$result is proved similarly.

\section{REFERENCES}

[HKLDW17] Thomas A. Hulse, Chan Ieong Kuan, David Lowry-Duda, and Alexander Walker. The second moment of sums of coefficients of cusp forms. Journal of Number Theory, 173:304-331, 2017.

[Ing90] A. E. Ingham. The distribution of prime numbers. Cambridge Mathematical Library. Cambridge University Press, Cambridge, 1990. Reprint of the 1932 original, With a foreword by R. C. Vaughan. 